\title{
Structural Insights into How Protein-Protein Interaction Modulates the Action of MEK Inhibitors
}

Zaigham Khan ${ }^{1}$, William Marsiglia ${ }^{2}$, Arthur Chow ${ }^{3}$, Alex Scopton ${ }^{3}$ and Arvin Dar $^{3}$

${ }^{1}$ Mount Sinai School of Medicine, New York, United States, Fort Lee, New Jersey, United States, ${ }^{2}$ Mount Sinai School of Medicine, New York, United States, New Jersey, United States, ${ }^{3}$ Mount Sinai School of Medicine, New York, United States, United States

The mitogen-activated protein kinase (MAPK/ERK Kinase) MEK is a shared effector of KRAS and BRAF; the most frequently mutated oncogene and protein kinase, respectively, across all human cancers. As such, MEK has long been pursued as a drug target in oncology, and more recently in immunotherapy and aging. At the cellular and molecular levels, MEK interacts with Raf family members including ARAF, BRAF, and CRAF kinases, and KSR1 and KSR2 pseudokinases. The expression level, localization, and interaction of MEK and RAF family members modulate the strength of MAP kinase signaling, and also presumably the pharmacology of the inhibitors. Several MEK inhibitors (MEKi) are approved for the treatment of various malignancies, and trametinib is the first and most successful MEKi. How trametinib occupies MEK allosteric pocket is not yet known. Recently we reported the X-ray crystal structures of MEK bound to scaffold KSR in complex with several MEKi, including clinical drug trametinib (1). In this physiological MEK-KSR complex, the protein-protein interaction remodeled the prototypical MEKi allosteric pocket of MEK, whereby the helix-G of KSR forms the border of the MEK allosteric pocket. Convincingly, trametinib is the only MEKi to extend to the MEK-KSR interface, and directly coordinates with KSR helix-G. Under the influence of MEK-KSR interaction, the residence time of trametinib is 3fold increased. Our structures led us design trametiglue that overcomes the adaptive resistance to MEK inhibition through enhanced interfacial binding. Our structural analysis is the first one to report the binding of MEKi to the MEK complexes, and suggest the role of MEK interaction partner on the kinetics of MEKi. RAF kinases are the direct activators of the MEK, our next goal to extend our analysis to RAF-bound MEK. We have recently solved the structure of BRAF-bound MEK in complex with several MEKi. We noticed pronounced structural differences between KSR-bound MEK and BRAF-bound MEK that may explain the altered kinetic parameters of MEKi. our structure-function analysis of MEK complexes will enhance our understanding on the role of MEK binding partners on the pharmacology of the MEKi, and will allow us to design novel drugs that may target the higher-order signaling complex.

Z Khan, A Real, W Marsiglia et al. Structural basis for the action of the drug trametinib at KSR-bound MEK. Nature; 588: 509-514 (2020) [PMID: 32927473] 


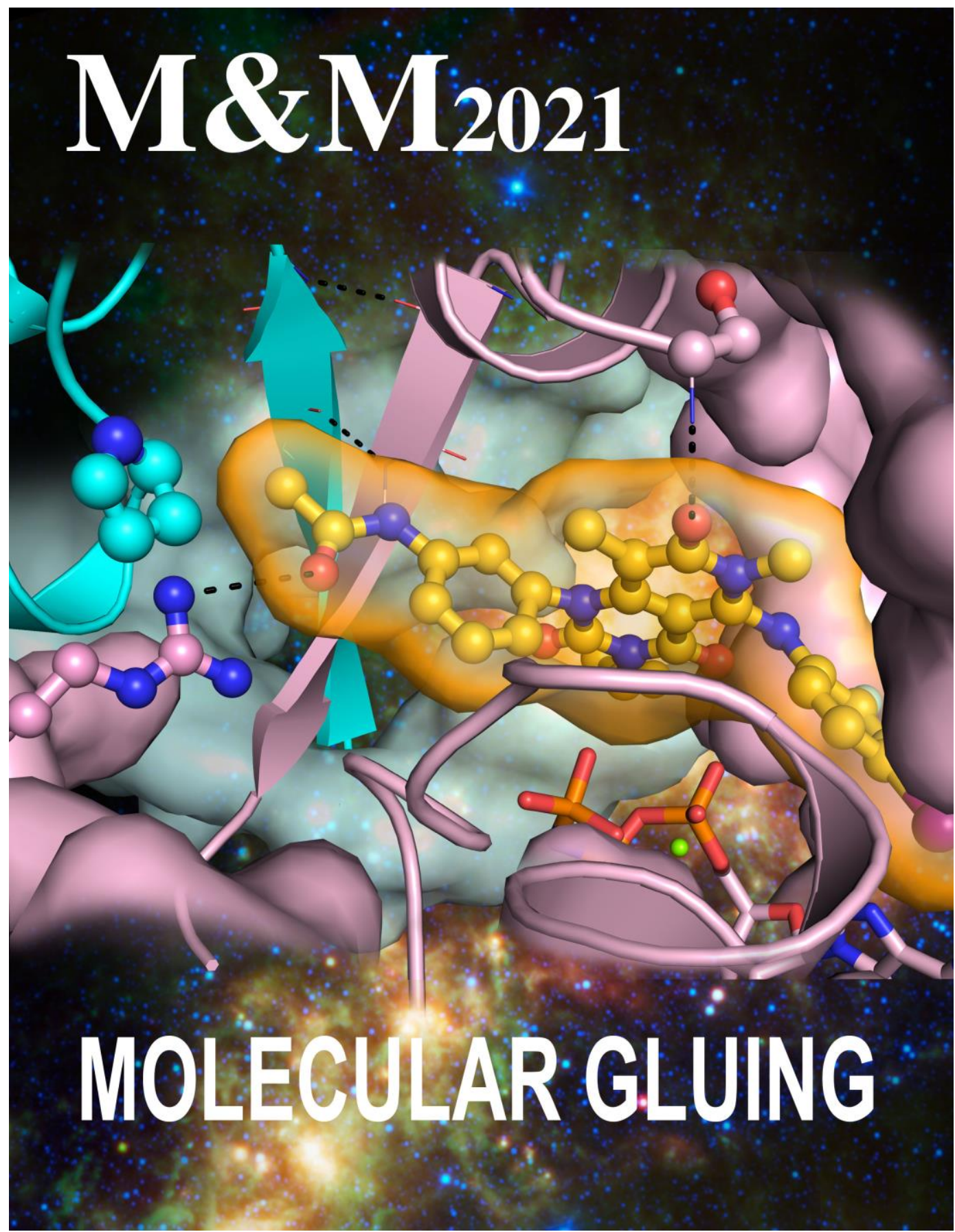

Figure 1. Molecular Gluing:FDA approved MEK inhibitor Trametinib binds MEK, and extends towards, and interacts with the protein binding partner, Kinase Suppressor of Ras (KSR) - a pseudokinase of RAF family. MEK-Trametinib-KSR mimic a gluing phenomenon that results in increased affinity and on-target retention time of trametinib. 


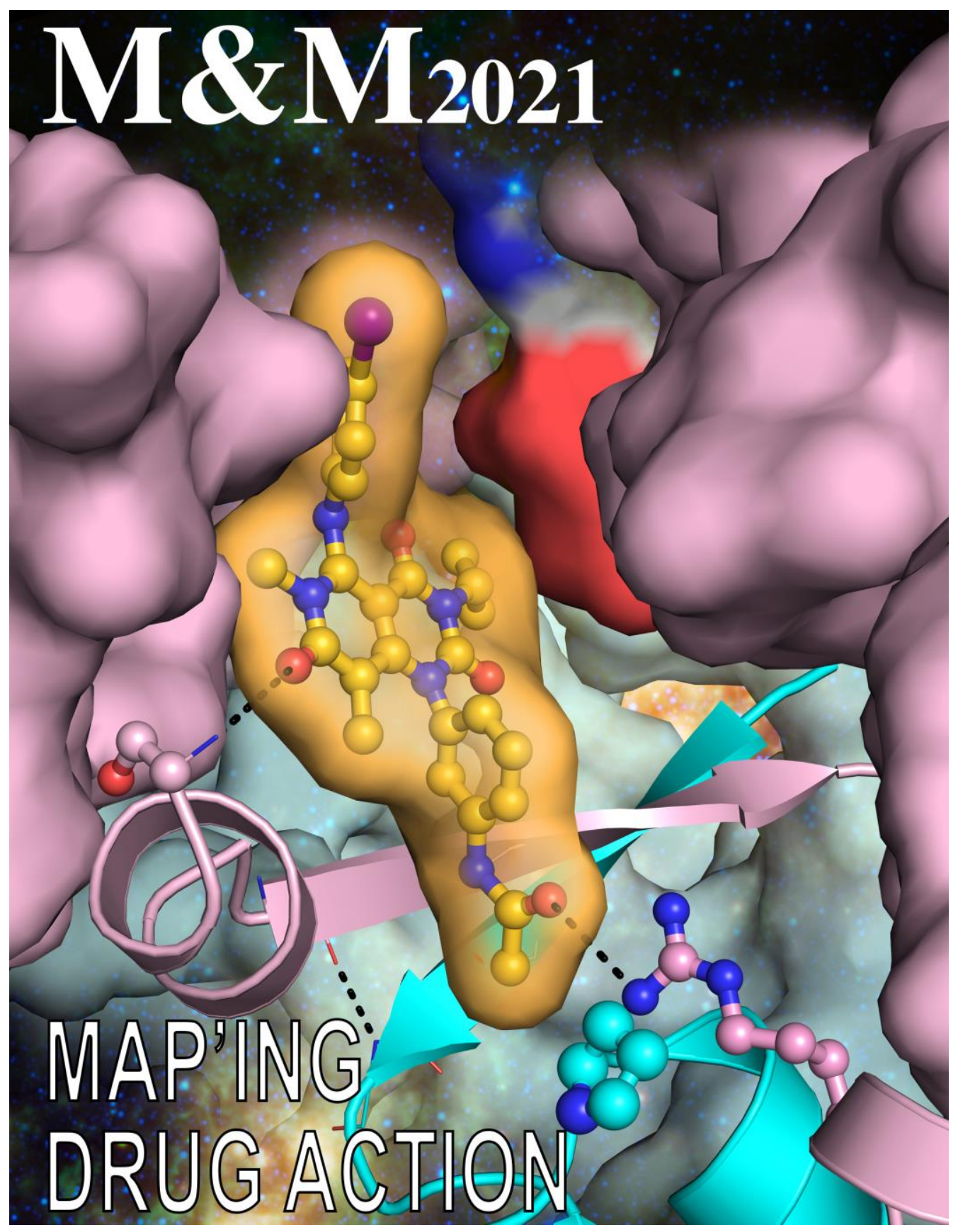

Figure 2. Map-ing the drug action:Trametinib is the first and most successful FDA-approved inhibitor that binds MEK, and inhibits the action of MAPK/ERK. The X-ray crystal structure of KSR-bound MEK in complex with trametinib revealed the binding mode where trametinib binds MEK, and owing to its size and geometrical constraints, projects towards and interacts KSR pseudokinase. This is the first structure of trametinib bound to its target kinase, and this is also the first structure to show that pseudokinase is a co-receptor for an FDAapproved drug.

\section{References}

1. Zaigham Khan et al. Structural basis for the action of the drug trametinib at KSR-bound MEK. Nature; 588: 509-514 (2020) [PMID: 32927473] 